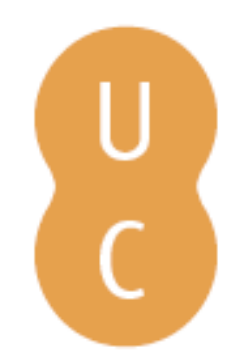

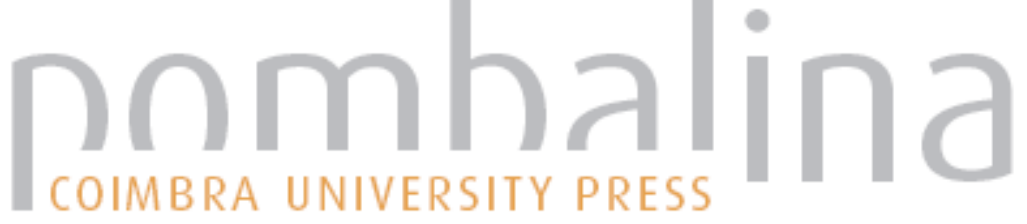

\section{Estudo do contacto de línguas em ambiente urbano}

Autor(es): $\quad$ Corrêa-Cardoso, João Nuno

Publicado por: Imprensa da Universidade de Coimbra

URL

persistente:

URI:http://hdl.handle.net/10316.2/39995

DOI:

DOI:https://doi.org/10.14195/978-989-26-1115-0_3

Accessed : $\quad$ 26-Apr-2023 11:30:12

A navegação consulta e descarregamento dos títulos inseridos nas Bibliotecas Digitais UC Digitalis, UC Pombalina e UC Impactum, pressupõem a aceitação plena e sem reservas dos Termos e Condições de Uso destas Bibliotecas Digitais, disponíveis em https://digitalis.uc.pt/pt-pt/termos.

Conforme exposto nos referidos Termos e Condições de Uso, o descarregamento de títulos de acesso restrito requer uma licença válida de autorização devendo o utilizador aceder ao(s) documento(s) a partir de um endereço de IP da instituição detentora da supramencionada licença.

Ao utilizador é apenas permitido o descarregamento para uso pessoal, pelo que o emprego do(s) título(s) descarregado(s) para outro fim, designadamente comercial, carece de autorização do respetivo autor ou editor da obra.

Na medida em que todas as obras da UC Digitalis se encontram protegidas pelo Código do Direito de Autor e Direitos Conexos e demais legislação aplicável, toda a cópia, parcial ou total, deste documento, nos casos em que é legalmente admitida, deverá conter ou fazer-se acompanhar por este aviso. 


\section{JOÃO CORRÊA-CARDOSO MARIA DO CÉU FIALHO}

(Coordenadores)

\section{A LINGUAGEM NA PÓLIS}

IMPRENSA DA UNIVERSIDADE DE COIMBRA COIMBRA UNIVERSITY PRESS
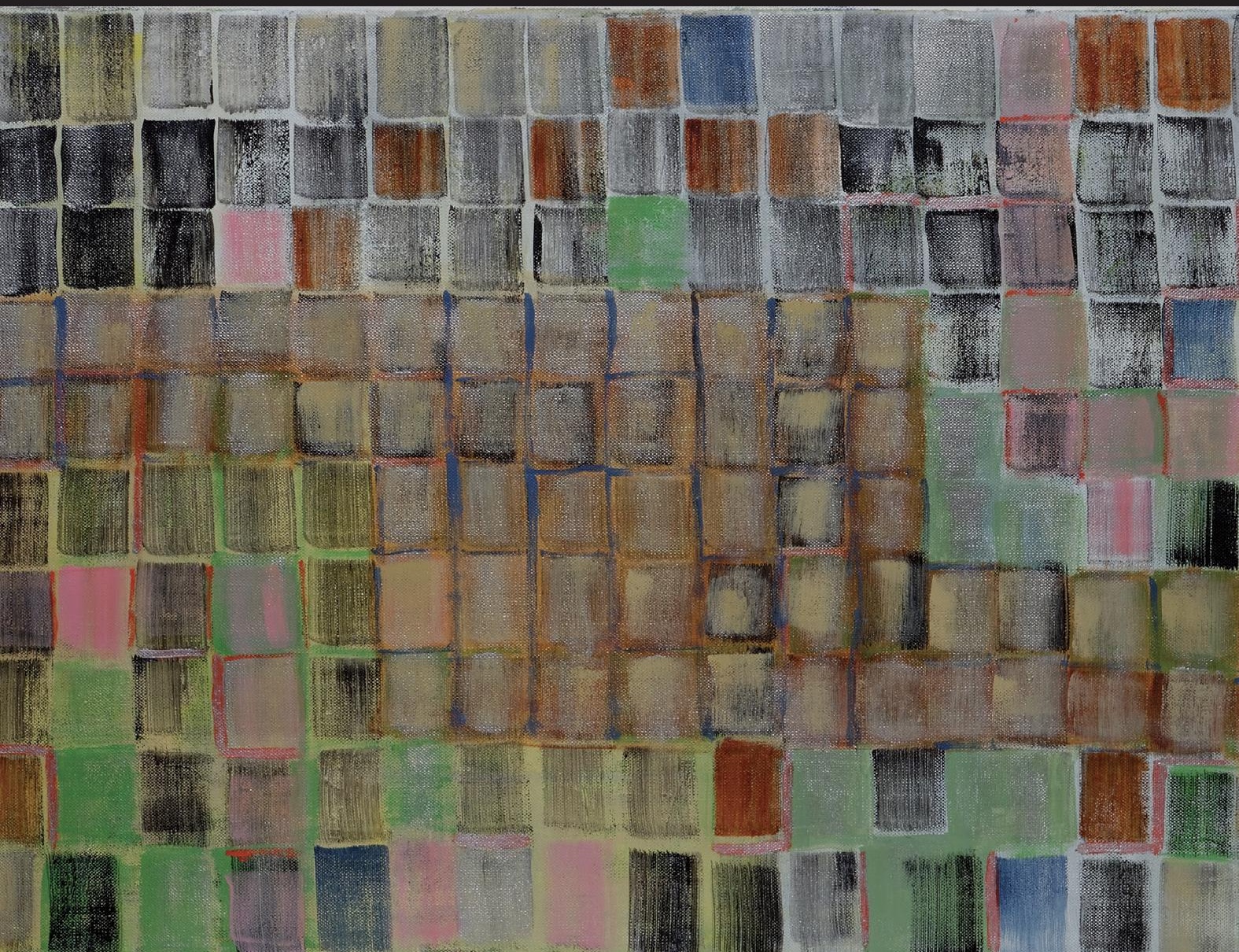


\section{ESTUDO DO CONTACTO DE LÍNGUAS \\ EMAMBI E NTE URBANO}

João Nuno Corrêa-Cardoso

Universidade de Coimbra cardo@ci.uc.pt

Resumo: Reflexões em torno do contacto de línguas em ambientes urbanos contemporâneos. Abordagem das novas metodologias exigidas pelas especificidades dos trabalhos de campo. A figura do linguista de contacto. Palavras chave: sociolinguística urbana de contacto; trabalho de campo nas cidades europeias contemporâneas; línguas maioritárias e minoritárias; desaparecimento de línguas.

Abstract: Reflections on urban language contacts. Study of field work trends and new methodological approaches required by the contemporary european urban contexts. The contact linguist profile.

Keywords: urban sociolinguistics; field work at contemporary european cities; majority and minority languages; language loss.

\section{Reflexões iniciais}

"The cities of Europe metamorphose rapidly. (...) The flux between the city and its inhabitants is a site of ferocious visual tension, with imageries generated that collapse and reformulate 
the perception of the city, its languages, its societies, its nationalities, its cultures. The cities have never possessed unity, and now the multiplicity of voices passing between the transforming city and the transforming individual creates an utter fragmentation.»1

1. A macroeconomia europeia aponta para a existência de dois grandes eixos que cruzam o continente. O primeiro é o do desenvolvimento urbano, de sentido norte-sul, cujos extremos são o Reino Unido e a Itália. O segundo é o da colonização industrial, de orientação oeste-leste, fixando-se os pólos, por um lado, em Inglaterra, no Benelux e na Alemanha e, por outro, na Ucrânia. A partir destas informações, podemos dizer que, de Londres a Milão, se desenha um arco que, hoje em dia, compreende a maioria das capitais económicas e dos centros financeiros da Europa.

2. De um modo global, o conjunto da população europeia deve ser considerado urbano quer pelos modos de vida e de pensar quer, também, pelas formas de comunicar. Mas se as taxas de urbanização da Europa são relativamente elevadas, há todavia - adotando o modelo da aglomeração, utilizado ultimamente por quem se dedica a estudos urbanísticos - zonas do espaço europeu em que a distribuição das pessoas pelas cidades não é equilibrada e são justamente as periferias que exibem os índices mais baixos de concentração urbana. Como exemplos ilustrativos, os estudos apontam, consensualmente e com regularidade desde 1990, para Portugal, a Irlanda, a Noruega e ainda para os países inscritos na instável teia geopolítica dos Balcãs.

3. No âmbito destas condições gerais, compreende-se que o fenómeno da mobilidade dos europeus - motivado por um número

${ }^{1}$ Stephen Barber (1995) Fragments of the European City. London (Reaktion Books, Ltd), p. 9. 
ilimitado de causas - os conduza quase sempre para cidades, locais que, por instituição histórico-cultural dos seus atributos reais e das suas propriedades simbólicas, têm por vocação acolher muitos e diferenciados atores da existência comunitária. Quando se registam saídas para o estrangeiro com a necessidade de se assegurarem as premissas mais basilares da dignidade humana, as urbes-destino destas populações desfavorecidas inscrevem-se na intersecção dos eixos de desenvolvimento acabados de referir.

4. Através destes movimentos migratórios inter-étnicos dão-se, em plena cidade, os encontros de línguas e de culturas que vão favorecendo, em tempos e a ritmos desiguais, a convivência intercultural e as inevitáveis transformações linguístico-culturais, inerentes a todos os atos manifestativos do homo urbanus, no decurso do conhecimento - que devia ser cada vez mais profundo - do Outro. O exame sociolinguístico de tais ambientes de comunicação plural deve ser empreendido na mais ampla reflexão interdisciplinar porque a natureza do objeto seleccionado é, como sabemos, multifacetada.

\section{Coordenadas do estudo sociolinguístico do contacto de lín- guas na cidade}

"Nothing exists of the city but duplication, repetition and prolongation, except for what is put there by the eye of the spectator, intercepted and visually substantiated. And with the falling of the eye upon the city comes the massive movement, gesture, flux of physical incorporation of the city, that gives to the city its psychosis of imagery and language (...) its (...) fragmentation, transformation.» ${ }^{2}$

2 Idem, ibidem, p. 107. 
5. Foi o desenvolvimento da linguística de contacto no âmbito das linguas minoritárias, nacionais e étnicas, bem como no das línguas das comunidades da diáspora, que deslocou as atenções para o contacto de línguas em meios urbanos. Não foi casual, então, o facto de a linguística urbana de contacto ter despertado no pós-guerra e ter vindo a registar, ultimamente, um desenvolvimento considerável.

Reconhecido o espaço linguístico urbano fragmentário, cada pesquisa terá de ser única e completar-se, apelando à pluridisciplinaridade: têm de se conhecer os parâmetros sociopsicológicos e socioculturais que afloram nas tensões co-presentes e que merecem o manuseamento científico. É a gramática da variação do atual mosaico linguístico urbano que exige uma análise da manutenção/discriminação das línguas em contacto e a explicitação desse devir, qualquer que ele seja. Assim, as investigações complementares elucidarão, por exemplo, o modo da re-afirmação cultural e (sócio-)linguística, a reconstrução das identidades e as formas de comunicação inter-grupal; a aceitação ou a recusa de aculturação, ou ainda outros aspetos dos complexos atitudinais. É também imprescindível conhecer as disposições políticas sobre a gestão linguística e intercultural nos Estados plurilingues.

6. A cidade, como instância pós-moderna, é a arena privilegiada dos encontros e dos desencontros das línguas e das culturas do bomem urbano que nela se instala e se diversifica - em cada momento diferenciado - através das comunhões simbólicas. A expressão dos aspetos polifacetados da tumultuosa vida em comum encontra, como seria de esperar, na palavra o veículo primordial dessa participação, a um mesmo tempo individual e coletiva, próxima e distante, objetiva e subjetiva, real e ficcionada, mas sempre indispensável aos mecanismos definitórios do Eu, do Outro, de Nós e dos não-Nós.

O progresso poderá aniquilar os traços fundamentais da dimensão humana que motivaram, desde muito cedo, a reunião do homo 
loquens nos lugares públicos, comprometendo-se, dessa forma, a possibilidade que cada um de nós deve ter, na cidade, de afirmação da liberdade pessoal e de gestão do próprio destino. Por tudo isto, no recente discurso filosófico defende-se o retorno à Pólis como um imperativo global e a tentativa de se substituir o desencontro humano - promovido modelarmente pela Telepolis - pelo encontro interpessoal, sustentado pela razão pática.

7. Com o encontro das culturas e a respetiva verbalização polifónica instaura-se, de forma premente, o plurilinguismo nas aglomerações urbanas cujas características e funcionalidades acompanham, por um lado, o ritmo de crescimento das cidades, da mobilidade social e cultural dos habitantes nativos e dos de adoção (antiga ou recente) e, por outro, se associam igualmente ao significado dos contactos interétnicos e às tensões relacionadas com as tentativas de domínio dos múltiplos espaços de circulação e de convivência citadinas.

Assim, qualquer aproximação científica da cena sociolinguística urbana deve explicitar a estruturação linguística da comunidade plural segundo a importância dos chamados fatores de variação que operam em aglomerados compósitos deste género e cruzar tais informações com outros dados fornecidos quer pela história particular (recuada ou próxima) das cidades, quer pelas disposições políticas que legitimam os estatutos linguísticos privilegiados das línguas dominantes, quer pelas raízes da constituição demográfica urbana quer, ainda, pelos efeitos psicológicos e ecológicos atuais da centralidade do ambiente na urbe.

8. É necessário contextualizar o contacto linguístico seja em termos materiais - observando as premissas demográficas e situacionais das práticas discursivas, por exemplo -, seja em termos culturais - analisando os percursos históricos, económicos, sociológicos e psicológicos das comunidades envolvidas, bem como as respetivas 
construções ideológicas e atitudinais, subjacentes aos comportamentos individuais e grupais - porque é, justamente, de todos estes elementos extralinguísticos que decorre, de facto, uma maior ou menor rejeição de tudo o que é sentido como sendo diferente ou, se quisermos, é deles que nascem os conflitos linguísticos (e todos os outros atritos) mais ou menos agudos.

Nunca esquecendo, porém, que o movimento de integração - voluntária ou forçada - se vive a ritmos distintos, poderemos definir a morte do sistema minoritário como uma das mais dramáticas consequências dos conflitos linguísticos que, desta forma, se vão superando. O fenómeno do suicídio linguístico verifica-se quando os dois sistemas são muito próximos, adotando aquele que se encontra na posição de dominado muitos elementos dos vários planos da língua dominante. $\mathrm{O}$ fenómeno do assassínio linguístico regista-se com a crescente redução dos domínios em que se atualizava a língua fraca, remetendo-se para o acentuado esquecimento o conhecimento linguístico (ativo e passivo) que dela possuíam os sujeitos falantes minoritários que, entretanto, deixam de possuir a qualidade de interlocutores bilingues para adquirirem, gradualmente, o estatuto de monolingues na língua forte.

Todavia, no contexto da emigração e, portanto, no caso das chamadas minorias recentes - cuja relação física e institucional com o território do new country é, numa primeira fase, sempre difícil - as fragilidades acentuam-se; e os ditos processos, conducentes a uma eficaz assimilação linguístico-cultural, podem vir a revelar-se ainda mais rápidos do que os próprios sujeitos falantes da língua em desvantagem suspeitariam. Tais mecanismos tanto são exógenos como são endógenos à comunidade (recém-chegada ou com uma breve história de fixação), e atuarão de forma diferenciada, de acordo com as características particulares de cada situação de encontro de linguas e de culturas. 
9. Partindo das grandes linhas gerais sugeridas para a observação do fenómeno do contacto, tenhamos em consideração, agora, items imprescindíveis para a avaliação das condições de erosão sofrida por qualquer língua emigrada.

Do ponto de vista estritamente linguístico, deve o investigador tentar determinar o grau de domínio da norma na língua de partida. Da plataforma sociológica, poderá o pesquisador - segundo os objetivos da sua análise - destacar aspetos tão variados como, por exemplo, a) o tipo do fluxo migratório, b) a distribuição setorial do movimento de partida, c) o nível sociocultural dos seus atores, d) a intensidade do sentimento de pertença ao grupo, e) a idade de chegada ao país recetor, f) o tipo de fixação no país de acolhimento, g) o lapso temporal de permanência e h) o grau de proximidade cultural e religiosa em relação à comunidade linguística recetora.

Como se pode concluir do quadro traçado, os eixos de abordagem das populações-alvo são duais - por conciliarem em si aspetos de natureza linguística e aspetos decorrentes da dimensão extralinguística - e o poder explicativo de cada um deles dependerá da sua adaptação ao perfil da área metropolitana selecionada e aos contornos dos grupos minoritários em causa. Ora, na conjuntura atual do plurilinguismo e da interculturalidade, a sociolinguistica urbana de contacto, desenvolvida em ambiente de emigrados estrangeiros, transporta - para o debate geral sobre a investigação linguística comunitária urbana - o imperativo de se estudar a petite minorité, na qualidade de zona humana de contacto interlectal mais reduzido. A este nível reticular, o estudo dos fenómenos da interação e da comunicação entre indivíduos de culturas e de línguas diferentes parece favorecer uma análise, cada vez menos opaca, da variação participativa nos múltiplos planos da atividade dialogal.

De acordo com as coordenadas assim definidas, o trabalho de campo sobre os efeitos da centralidade urbana nos contactos de línguas e a caracterização técnica dos comportamentos linguísticos 
observados nunca devem deixar de se nortear, quando se trata do relacionamento multifacetado entre uma lingua forte e uma ou mais línguas débeis, pelos objetivos que passo a enumerar:

$\left.1 .^{\circ}\right)$ deteção das estruturas que asseguram o domínio de uma língua sobre todas as outras;

$\left.2 .^{\circ}\right)$ definição da(s) (in)consistência(s) das minorias linguísticas;

$\left.3 .^{\circ}\right)$ explicitação dos padrões e dos significados dos contactos interétnicos;

$4^{\circ} .^{\circ}$ interpretação dos estados de afeto(s) da(s) língua(s) minoritária(s).

10. Nesta linha de pensamento - que obriga a uma rápida ação transversal - dignificar-se-á, de modo indubitável, a tradição humanista e humanitária e caberá, em primeiro lugar, às próprias famílias e à instituição Escola o papel central no crescimento do respeito mútuo e da paz de que nós, também neste domínio específico, tanto carecemos.

A democraticidade na co-presença deverá, de facto, iniciar-se na célula familiar e nas instituições educativas onde os responsáveis pela formação harmoniosa do cidadão europeu empreenderão um exercício constante de reflexão sobre as suas atitudes e comportamentos linguísticos de maneira a transmitir, às crianças e aos jovens, a ideia de plurilinguismo em que vivem e em que, legitimamente, se podem expressar sem os preconceitos que têm alimentado as tensões entre os que teimosamente desconfiam da diferença e os incompreendidos.

A persistência das clivagens favorecerá a continuação das desigualdades sociais com consequências devastadoras para todos aqueles que não se podem defender - em especial as crianças - dado não possuírem os instrumentos (nem a força) para uma resposta adequada necessária à alteração dos preconceitos do generalizado e desfavorável senso-comum. 
11. O investigador, movendo-se nesse espaço linguístico urbano, detetará não só a emergência de um núcleo linguístico, como a viabilidade de gerir as diferenças - o que se baseia, por paradoxal que pareça, em tratamentos diferentes para atingir uma igualdade de oportunidades. Perceber isso e realizá-lo é fruto de uma ampla visão e de qualidades humanas superiores. Assim, o maior incentivo do sociolinguista urbano será a busca do plurilinguismo como um triunfo do espírito humano. O primeiro passo cabe à comunidade linguística de prestígio. Depois, as diretivas dos Estados plurilingues indicarão as abordagens adequadas de um plurilinguismo concentrado no perímetro urbano.

A responsabilidade do linguista do contacto é, então, também tanto maior quanto mais dele se exige um papel político que inclui a diplomacia, para explicar como, em presença de diversas línguas e culturas, se pode gerir o encontro de valores comunitários; isto para além do seu papel de cientista que, tendo analisado a cidade nas suas distintas faces e reverberações, deve ajudá-la a ultrapassar os conflitos.

\section{Reflexões conclusivas}

"The trajectory of language through the city is shadowed and countered by its erosion - even the most uniquely intense, vertiginous projection of language (...) will collapse in its act of realization, and lapse into silence." 3

12. Apesar de toda a política linguística ser enquadrada por preceitos legais, na prática, é numa complexa arena plurilingue que se joga o futuro de uma língua minoritária. Assim, o estudo do

3 Idem, Ibidem, p. 85. 
contacto linguístico deve ser material e culturalmente contextualizado, atendendo às ideias e atitudes subjacentes ao comportamento individual e grupal e deve basear-se no conhecimento minucioso das comunidades em questão: porque há conflitos linguísticos e a sua consequência mais grave será a morte do sistema linguístico minoritário. Este óbito é, afinal, e como refere Louis-Jean Calvet, uma resolução possível dos conflitos linguísticos existentes. Quer isto dizer que uma evolução deste tipo pode ser uma estratégia dos poderes instituídos para, a prazo (in)certo e discretamente, reforçar a sua estabilidade. Pierre Bourdieu complementa esta ideia, lembrando a importância da coesão no mercado linguístico que, ao valorizar a unificação dos produtos verbais, garante o domínio linguístico do sistema mais forte. A população minoritária deixa de ter capacidade para ser bilingue e, gradualmente, vai adquirindo o estatuto de monolingue na língua forte. No caso das minorias recentes, psicológica e culturalmente frágeis, ainda mais rápida é a erosão linguística. No estado atual das pesquisas, estamos, de facto, muito longe da visão otimista que concebe pacífica a urbe plurilingue e pluricultural.

\section{Bibliografia}

Ali, Aydin Mehmet (1989), The Turkish Community in Britain: Some Comments and Observations on the Immigration Patterns, and the Legal and Social Position. In: Language Issues. Vol. 3, Number 1, Spring/Summer.

Alvar, Manuel / López Morales, Humberto (1978), Estudios sociolingüísticos. Colecção Cuadernos de Lingüística. $\mathrm{n}^{\circ}$ 5. Primera edición. México (Universidad Nacional Autónoma de México - Instituto de Investigaciones Filológicas - Centro de Lingüística Hispánica).

Alvarez Reyes, Juan Antonio (1997), La forma del habitar colectivo: el poder en (la) forma. In Desde la ciudad. Huesca: arte y naturaleza. Actas del IV Curso. Huesca (Diputación de Huesca).

André, João Maria (1999), Fénix e as cinzas do século. Seis contrapropostas para o próximo milénio. In Pensamento e afectividade. Coleção Caminhos. Vol. I, Coimbra (Quarteto Editora), p. 139-170.

Ambrose, John E. / Williams, Colin H. (1990), On the Spatial Definition of 'Minority': Scale as an Influence on the Geolinguistic Analysis of Welsh. In Minority Languages 
Today. A Selection from the Papers read at the First International Conference on Minority Languages held at Glasgow University from 8 to 13 September 1980. Edited by Einar Haugen, J. Derrick McClure, Derick Thomson. Edinburgh (Edinburgh University Press).

Apoio da Comissão Europeia às acções de promoção e de salvaguarda das línguas e culturas regionais ou minoritárias. (97/C 178/04). Texto relevante para efeitos do EEE. In Jornal Oficial das Comunidades Europeias. Edição em língua portuguesa. C178. $40 .^{\circ}$ ano. Luxemburgo (Serviço das Publicações Oficiais das Comunidades Europeias), 12 de Junho de 1997.

Apoio da Comissão Europeia às acções de promoção e protecção das línguas regionais ou minoritárias. In Jornal Oficial das Comunidades Europeias. Edição em língua portuguesa. C 125/14. Luxemburgo (Serviço das Publicações Oficiais das Comunidades Europeias), 6 de Maio de 1999.

Arroteia, Jorge Carvalho / Doudin, Pierre-André (1998), Trajectórias sociais e culturais de jovens portugueses no espaço europeu. Questões multiculturais e de integração. Aveiro (Universidade de Aveiro).

Ascher, François (1995), Métapolis ou l'avenir des villes. Paris (Editions Odile Jacob).

Bakus, Ad (1996), Two in One. Bilingual Speech of Turkish Immigrants in The Netherlands. Colecção Studies in Multilingualism. Vol. 1. René APPEL, Guus Extra, Koen Jaspaert, Ludo Verhoeven (Editors). Tilburg (Tilburg University Press).

Baker, Philip / Eversley, John (2000), Introducing the Languages of London Project. In Multilingual Capital. The Languages of London's Schoolchildren and their Relevance to Economic, Social and Educational Policies. Preface by The Right Honourable The Lord Mayor Alderman Clive Martin. Edited by Philip Baker and John Eversley. London (Battlebridge Publications).

Baker, Philip /Mohieldeen, Yasir (2000), The Languages of London's Schoolchildren. In Multilingual Capital. The Languages of London's Schoolchildren and their Relevance to Economic, Social and Educational Policies. Preface by The Right Honourable The Lord Mayor Bannick, Christian John (1917), Portuguese Immigration to the United States: Its Distribution and Status. University of California.

Barou, Jacques (2001), Europe, terre d'immigration. Flux migratoires et intégration. Collection Transeurope, dirigée par Jean-Marie MARTIN. Grenoble (Presses Universitaires de Grenoble).

Barreto, António (1997), Tempo de mudança. 2. ${ }^{a}$ edição. Colecção Antropos. Vol. 7. Lisboa (Relógio D’Água Editores).

Bastardas I Boada, Albert (1986), "Bilingüització de la segona generació immigrant: realitat i factors del procés a Vilafranca del Penedès." In Treballs de sociolingüística catalana. N. ${ }^{\circ} 6$.

Bauer, Roland (1999), Sprachsoziologische Studien zur Mehrsprachigkeit im Aostatal: mit besonderer Berücksichtigung der externen Sprachgeschichte. Beihefte zur Zeitschrift für romanische Philologie. Vol. 296. Tübingen (Niemeyer).

Beaujeu-Garnier, Jacqueline (1997) Geografia urbana, $2^{a}$ ed. Tradução de Raquel Soeiro de Brito. Lisboa (Fundação Calouste Gulbenkian).

Bediafi, C., Social Motivations for Code-Switching Amongst Urban Tunisians. Documento obtido na Internet, em 22 de Outubro de 1998. 
Beirão, Delfina (1999), Les portugais du Luxembourg. Des familles racontent leur vie. Préface de Fernand Fehlen. Collection Migrations et Changements. Vol. 52. Paris, Montréal (Ciemi - L'Harmattan).

Blas Arroyo, José Luis (1993), La interferencia lingüística en Valencia (dirección:

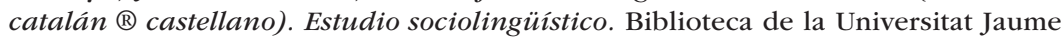
I. València (Publicacions de la Universitat Jaume I).

Borrego Nieto, Julio (1981), Sociolingüística rural. Investigación en Villadepera de Sayago. Colecção Acta Salmanticensia. Filosofía y Letras. N. ${ }^{\circ}$ 120. Studia Philologica Salmanticensia. Anejos. Estudios. N. ${ }^{\circ}$ 3. Salamanca (Ediciones Universidad de Salamanca).

Bortoni, Stella Maris (1989), A migração rural-urbana no Brasil: uma análise sociolingüistica. In Fotografias sociolingüísticas. Colecção Linguagem/Crítica. Fernando Tarallo (Org.). Prefácio de Ataliba T. de Castilho. Apresentação de Fernando Tarallo. Campinas (Pontes Editores - Editora da Universidade Estadual de Campinas).

Bourdieu, Pierre (1994), Le marché linguistique. In Questions de sociologie. Édition augmentée d'un index. Paris (Les Éditions de Minuit).

Boyer, Henri (1996), Éléments de sociolinguistique. Langue, communication et société. Avec la collaboration de Gloria Bayo. Deuxième édition revue et corrigée. Paris (Dunod).

Boyer, Henri (1996), Les domaines de la sociolinguistique. In Sociolinguistique. Territoire et objets. Sous la direction de H. Boyer. Colecção Actualités en Sciences Sociales. Textes de base en Sciences Sociales. Collection dirigée par Jean-Claude Deschamps et Marie-Noëlle Schurmans. Lausanne, Paris (Delachaux et Niestlé).

Boyer, Henri (2001), Introduction à la sociolinguistique. Colecção Les topos. Paris (Dunod).

Brandhove, Ulla (1997), Studien zum Dialektstatus in sechs Marburger Stadtteilen: soziolinguistische und diachron-kontrastive Analysen. Colecção Deutsche Sprache und Literatur. Bd. 1642. Europäische Hochschulschriften: Reihe 1. Frankfurt am Main, Berlin (Lang).

Burgel Guy (1995), La ville aujourd'hui. Collection Pluriel dirigée par Pierre Vallaud. Paris (Hachette).

Calero Fernández, María Ángeles (1993), Estudio sociolingüístico del habla de Toledo: segmentos fonológicos $-/ s / y-/ j /-$. Prólogo de Humberto LÓPEZ MORALES. $1 .^{a}$ edición. Collecció El Fil d'Ariadna. Direcció: Àngels Santa. Sèrie Lingüística. Vol. 16. Lleida (Publicacions de la Universitat de Lleida - Fundació Pública - Institut d'Estudis Llerdencs de la Diputació de Lleida i el Departament de Cultura de la Paeria - Ajuntament de Lleida - Pagès editors).

Callou, Dinah Maria I. / Costa, Maria Cristina Rigoni (1996), A linguagem do Rio de Janeiro. Manuscrito da Comunicação apresentada no XI Encontro Nacional da Associação Portuguesa de Linguística, realizado na Faculdade de Letras da Universidade de Lisboa entre 2 e 4 de Outubro de 1995, + 4 gráficos.

Calvet, Louis-Jean (1993), La sociolinguistique. 1ère édition. Colecção Que sais-je?, $\mathrm{n}^{\circ}$ 2731. Paris (Presses Universitaires de France).

(1994), Les voix de la ville. Introduction à la sociolinguistique urbaine. Colecção Essais Payot. Paris (Éditions Payot \& Rivages). 
Cardoso, João Nuno Paixão Corrêa (1997), A freguesia de Almalaguês par elle-même. Separ. da Revista Portuguesa de Filologia. Vol. XXI.

(1999), Sociolinguística rural. A freguesia de Almalaguês. Colecção Estudos. Vol. 27. Editor Fernando Mão De Ferro. Lisboa (Edições Colibri Faculdade de Letras da Universidade de Coimbra).

(2000), 'Wo meine Heimat ist, weiss ich nicht genau': aspectos da construção linguística da identidade em crianças portuguesas residentes em duas cidades alemãs. In: Actes du XXII Congrès International de Linguistique et de Philologie Romanes. Bruxelles, 23-29 juillet 1998. Publiés par Annick Englebert, Michel Pierrard, Laurence Rosier et Dan Van Raemdonck. Vol. III. Vivacité et diversité de la variation linguistique. Tübingen (Max Niemeyer Verlag).

(2011), Quando falo português sinto-me diferente, um pouco estrangeira. As atitudes linguísticas de um grupo infanto-juvenil cabo-verdiano. Separata da Miscelânea de Estudos em Homenagem a Maria Manuela Gouveia Delille. Vol. I / Band I. Coimbra (Faculdade de Letras da Universidade de Coimbra, Centro de Investigação em Estudos Germanísticos e MinervaCoimbra), p. 561-572.

(2011), Sociolinguística urbana de contacto. O português falado e escrito no Reino Unido. Presentación de Humberto LÓPEZ MORALES. Coleção Raiz do Tempo dirigida por Margarida Sobral NETO, Coimbra (Palimage).

Cardozo, Manoel da Silveira (1976), The Portuguese in America 590 B. C. - 1974. A Chronology and Fact Book. Ethnic Chronology Series. Number 22. New York (Oceana Publications, Inc.).

Carreira, Maria Helena de Araújo (1987), A língua portuguesa em situação de emigração (França): elementos para uma reflexão sociolinguística. In: Actas do Congresso sobre a situação actual da língua portuguesa no Mundo. Vol. 2. Lisboa, 1983. Lisboa (ICALP).

Carrel, Silvia (1994), Du droit individuel au droit collectif. L'utilisation des langues moins répandues dans les services publics. Preface de Dónall ó Riagáin. Colecção Langues Vivantes. Vol. 2. Bruxelles (Le Bureau Européen pour les Langues Moins Répandues).

Carta dos Direitos Fundamentais da União Europeia. Proclamação solene. In Jornal Oficial das Comunidades Europeias. (2000/C 364/01), 18. 12. 2000.

Certeau, Michel de (1993), La culture au pluriel. Nouvelle édition établie et présentée par Luce GIARD. Collection Points. Série Essais. Vol. 267. Paris (Christian Bourgois Éditeur - Éditions du Seuil).

Child Rights. United Nations Children's Fund - UNICEF. Documento obtido na Internet, em 23 de Outubro de 1998.

Cidade, cultura e globalização (1997). Carlos FORTUNA (Org.). Colecção Sociologias coordenada por Rui Pena Pires. Oeiras (Celta Editora).

CLYNE, Michael (1987), History of Research on Language Contact. In: Sociolinguistics. An International Handbook of the Science of Language and Society. Foreword by Hugo Steger and Herbert Ernst Wiegand. Preface by Ulrich Ammon, Norbert Dittmar, Klaus J. Mattheier. Edited by Ulrich Ammon, Norbert Dittmar, Klaus J. Mattheier. Vol. I. Berlin. New York (Walter de Gruyter). 
Clyne, Michael (2003), Dynamics of Language Contact. English and Immigrant Languages. Colecção Cambridge Approaches to Language Contact. General Editor: Salikoko S. Mufwene. Cambridge (Cambridge University Press).

Costa, António Firmino da (1999), Sociedade de bairro. Dinâmicas sociais da identidade cultural. Colecção Sociologias coordenada por Rui Pena Pires. Oeiras (Celta Editora).

D'Agostino, Mari (1995), Per un 'atlante urbano': alcune riflessioni. In: Percorsi di geografia linguistica. Idee per un atlante siciliano della cultura dialettale e dell'italiano regionale. A cura di Giovanni Ruffino. Vol. 1. Palermo.

D'Souza, Jean (1994), Characterizing a 'Sociolinguistic Area'. In: Orbis. Tome XXXVII.

Eckert, Eva (1988), "First-Generation American Czech: A Sociolinguistic Survey." In: Language Problems and Language Planning. Vol. 12, $\mathrm{n}^{\circ} 2$.

Edwards, Walter F. (1992), "Sociolinguistic Behavior in a Detroit Inner-City Black Neighborhood." In: Language in Society. Vol. 21, $\mathrm{n}^{\circ} 1$.

Enquesta metropolitana 1986. Condicions de vida i hàbits de la població de l'àrea metropolitana de Barcelona. Dirigida per María Jesús Izquierdo, Fausto Miguélez, Marina Subirats. Volum 20. Transmissió i coneixement de la llengua catalana a l'àrea metropolitana de Barcelona. Monografia a cura de Marina Subirats. Presentació de Jordi Borja. Proleg de Miquel Strubell i Trueta. Barcelona (Institut de Sociolingüística Catalana - Ministerio de Trabajo y Seguridad Social - INEM Barcelona - Bellaterra Universitat Autonoma de Barcelona - Centre pel Desenvolupament de l'Economia Social - Institut d'Estudis Metropolitans de Barcelona), 1991.

Esses, Victoria / Haddock, Geoffrey / Zanna, Mark P. (1993), Values, Stereotypes, and Emotions as Determinants of Intergroup Attitudes. In Affect, Cognition, and Stereotyping: Interactive Processes in Group Perception. Diane M. Mackie, David L. Hamilton (eds.). San Diego (Academic Press).

Esteva Fabregat, Claudi (1977), Aculturació lingüística d'immigrats a Barcelona. In: Treballs de sociolingüística catalana. $\mathrm{N}^{\circ} 1$.

Etxebarria Arostegui, Maitena (1985), Sociolingüística urbana: el habla de Bilbao. Colecção Filosofía y Letras. Vol. 165. Salamanca (Acta Salmanticensia).

Fennell, Desmond (1990), Can a Shrinking Linguistic Minority be Saved? Lessons from the Irish Experience. In Minority Languages Today. A Selection from the Papers read at the First International Conference on Minority Languages held at Glasgow University from 8 to 13 September 1980. Edited by Einar Haugen, J. Derrick McClure, Derick Thomson. Edinburgh (Edinburgh University Press).

Ferguson, Charles A. (1971), National Sociolinguistic Profiles Formulas. In: Language Structure and Language Use. Essays by Charles A. Ferguson. Selected and introduced by Anwar S. Dil. Colecção Language Science and National Development. A Series Sponsored by the Linguistic Research Group of Pakistan. General Editor Anwar S. Dil. Stanford (Stanford University Press).

Fontanella De Weinberg, Maria Beatriz (1979), La asimilación lingüística de los inmigrantes. Mantenimiento y cambio de lengua en el sudoeste bonaerense. Colecção Série Letras, Vol. III. Bahia Blanca (Departamento de Ciencias Sociales - Universidad Nacional del Sur). 
Forrest Alan (1998), The Politics of Language in the European Union. In: European Review. Vol. 6. N. ${ }^{\circ} 3$.

Fortuna, Carlos (1999), Identidades, percursos, paisagens culturais. Colecção Sociologias coordenada por Rui Pena PIRES. Oeiras (Celta Editora).

Franceschi, Temistocle (1970), Lingua e cultura di una comunità italiana in Costa Rica. Colecção Ricerche Costaricenci dirette da Emilio Peruzzi. Vol. I. Firenze (Valmartina Editore).

Geerts, Guido (1987), Research on Language Contact. In Sociolinguistics. An International Handbook of the Science of Language and Society. Foreword by Hugo Steger and Herbert Ernst Wiegand. Preface by Ulrich Ammon, Norbert Dittmar, Klaus J. Mattheier. Edited by Ulrich Ammon, Norbert Dittmar, Klaus J. Mattheier. Vol. I. Berlin. New York (Walter de Gruyter).

Gómez Molina, José R. (1998), Actitudes lingüísticas en una comunidad bilingüe y multilectal. Área metropolitana de Valencia. Prólogo de Antonio Briz. Cuadernos de Filología. Anejo XXVIII. València (Universitat de València).

Grafmeyer, Yves (1996), Sociologie urbaine. Collection Sociologie. Vol. 128. Paris (Éditions Nathan).

Guidelines for the Investigation of the Language of Inner-city pupils. Director: Harold Rosen. Research Officer: Tony Burgess. London (Institute of Education).

Haugen, Einar (1990), Language Fragmentation in Scandinavia: Revolt of the Minorities. In: Minority Languages Today. A Selection from the Papers read at the First International Conference on Minority Languages held at Glasgow University from 8 to 13 September 1980. Edited by Einar Haugen, J. Derrick McClure, Derick Thomson. Edinburgh (Edinburgh University Press).

Havrest, Latif (1998), Sprachpolitik, Sprachenrecht und Sprachplanung im geteilten Kurdenland. Wien (Passagen-Verlag).

Hoffmann, Fernand (1990), Triglossia in Luxemburg. In: Minority Languages Today. A Selection from the Papers read at the First International Conference on Minority Languages held at Glasgow University from 8 to 13 September 1980. Edited by Einar Haugen, J. Derrick McClure, Derick Thomson. Edinburgh (Edinburgh University Press).

Identidade europeia e multiculturalismo. Actas do Curso Intensivo, 26 de Fevereiro a 7 de Março de 2002. Maria Manuela Tavares Ribeiro (coord.). Colecção Estudos sobre a Europa. Vol. 2. Coimbra (Quarteto Editora).

Identités et droits des minorités culturelles et linguistiques (2000). Études réunies, sous la direction de Danièle Vazeilles, par Günter Behling, Emmanuelle Ferreol et Gabriel Preiss. Introduction par Patricia Caupert-Ouellet. Montpellier (Publications IDES et LASPEC - Université Paul-Valéry).

Jerab, N. (1988), L'arabe des maghrébins. Une langue, des langues. In: Vingt-cinq communautés linguistiques de la France. Tome 2. Les langues immigrées. Sous la direction de Geneviève Vermes. Collection Logiques Sociales dirigée par Dominique Desjeux. Paris (Editions L'Harmattan).

Jodelet, Denise (1997), Représentations sociales: un domaine en expansion. In; Les représentations sociales. $5^{\mathrm{e}}$ édition. Sous la direction de Denise Jodelet. Collection Sociologie d'aujourd'hui, dirigée par Georges Balandier. Paris (Presses Universitaires de France). 
Kallmeyer, Werner (1996), Plurilinguisme dans les agglomérations urbaines. In Kontaktlinguistik. Ein internationales Handbuch zeitgenössischer Forschung. Herausgegeben von Hans Goebl, Peter H. Nelde, 凶denek Starý. 1. Halbband. Berlin, New York (Walter de Gruyter).

Laborit, Henri (1973), El hombre y la ciudad. Primera edición. Traducción de Agustín Gil Lasierra. Barcelona (Editorial Kairós).

Labov, William (1966), The Social Stratification of English in New York City. Introductory Note by Alfred S. Hayes. Preface by William Labov. Washington, D. C. (Center for Applied Linguistics).

Labov, William (1973), The Effect of Social Mobility on Linguistic Behavior. In: Explorations in Sociolinguistics. $4^{\text {th }}$ edition. Edited by Stanley Lieberson. Introduction by Stanley Lieberson. Foreword by Herman Turk. Colecção Language Science Monographs. Vol. 44. Bloomington (Research Center for the Language Sciences - Indiana University).

Labov, William (1976), The Study of Language in its Social Context. In: Advances in the Sociology of Language. $2^{\text {nd }}$ edition. Edited by Joshua A. Fishman. Preface by Joshua A. Fishman. Vol. I. The Hague (Mouton \& Co., Printers).

Labov, William (1981), The Study of Nonstandard English. Foreword by A. Hood Roberts. Urbana (National Council of Teachers of English).

Lamiquiz, Vidal / Carbonero, Pedro (1987), Perfil sociolingüístico del sevillano culto. Colecção Cuadernos del I.D.R. Vol. 12. Sevilla (Instituto de Desarrollo Regional de la Universidad de Sevilla).

Lang, Henry R. (1887-1889), Falar português de New-Bedford. In: Revista Lusitana. Vol. I.

Languages in Contact and Conflict. Contrasting Experiences in the Netherlands and Belgium. Preface by Sue Wright. Edited by Sue Wright with Hellen Kelly. Clevedon, Bristol, Adelaide (Multilingual Matters Ltd), 1995.

Laponce, Jean A. (1987), Languages and their Territories. Translated from the French by Anthony Martin- Sperry. Toronto, Buffalo, London (University of Toronto Press).

L'école de Chicago. Naissance de l'écologie urbaine (1998). Textes traduits et présentés par Yves Grafmeyer et Isaac Joseph. Nouvelle édition. Série Champ urbain, dirigée par Isabelle Billiard. Aveyron (Editions Aubier).

Lepoutre, David (1997), Cour de banlieue. Codes, rites et langages. Paris (Éditions Odile Jacob).

Les expectatives d'ús, actituds i necessitats lingüístiques entre la població adulta de l'aglomeració urbana barcelonina (1984). Enquesta efectuada l'octubre de 1983. Direcció General de Política Lingüística. Presentació de Isidor Marí, Joan M. Romaní. Barcelona (Departament de Cultura de la Generalitat de Catalunya).

Les minorités en Europe. Droits linguistiques et droits de l'Homme (1992). Avec une carte des langues d'Europe hors texte. Sous la direction de Henri Giordan. Collection Librairie européenne des Idées. Paris (Éditions Kimé).

Les sociétés pluriculturelles (1994). Sous la direction de Gabriel Gosselin et Henri Ossebi. Collection Logiques Sociales dirigée par Dominique Desjeux et Bruno Pequignot. Paris (Éditions L'Harmattan). 
Lieberson, Stanley (1987), Language Barriers between Different Speech Communities/ International Problems of Communication. In: Sociolinguistics. An International Handbook of the Science of Language and Society. Foreword by Hugo Steger and Herbert Ernst Wiegand. Preface by Ulrich Ammon, Norbert Dittmar, Klaus J. Mattheier. Edited by Ulrich Ammon, Norbert Dittmar, Klaus J. Mattheier. Vol. I. Berlin. New York (Walter de Gruyter).

Linguistic Diversity in London Schools. An Investigation carried out in the English Department of the University of London Institute of Education. Questionnaire Information. Director: Harold Rosen. Research Officer: Tony Burgess. Assisted by: John Snodgrass, Jane Miller. Pas. Red. Ros. (O's).

López Morales, Humberto (1989), Indices de mortandad léxica en Puerto Rico: El Proyecto Malaret. In: Asomante. Investigaciones lingüísticas sobre el español de Puerto Rico. Realizadas en el Instituto de Lingüística de la Universidad de Puerto Rico. Edición especial. n ${ }^{\circ}$ 1-2, Vol. XXXVII, Año XXXVII.

(1989), Sociolingüística. Colecção Biblioteca Románica Hispánica dirigida por Dámaso Alonso. III. Manuales. Vol. 70. Madrid (Editorial Gredos).

(1998), La aventura del español en América. Colecção Ensayo y Pensamiento. Madrid (Editorial Espasa Calpe, S. A.).

(1994), Métodos de investigación lingüística. $1^{a}$ edición. Colecção Biblioteca Filológica. Director de la Colección: Ricardo de La Fuente Ballesteros. Vol. 1. Salamanca (Ediciones Colegio de España).

Lüdi, Georges (1990), Les migrants comme minorité linguistique en Europe. In: Sociolinguistica. Vol. 4. Minderheiten und Sprachkontakt.

Macaulay, Ronald K. S. (1997), Standards and Variation in Urban Speech. Examples from Lowland Scots. Colecção Varieties of English Around the World. General Editor: Edgar W. SCHNEIDER. General Series. Vol. 20. Amsterdam, Philadelphia (John Benjamins Publishing Company).

Madera, Mónica (1996), Speech Community. In: Kontaktlinguistik. Ein internationales Handbuch zeitgenössischer Forschung. Herausgegeben von Hans Goebl, Peter H. Nelde, Ždenek Starý. 1. Halbband. Berlin, New York (Walter de Gruyter).

Marconot, Jean-Marie (1990), Le français parlé dans un quartier HLM. In: Langue Française. Vol. 85.

Martínez Martín, Francisco Miguel (1983), Fonética y sociolingüística en la ciudad de Burgos. Prólogo de Antonio Quilis. Colecção Collectanea Phonetica. Vol. VIII. Editada bajo la dirección de Antonio Quilis. Madrid (Consejo Superior de Investigaciones Científicas - Instituto Miguel de Cervantes).

Martínez-Regeira, Ana Teresa (2001), Usos innovadores de estar en la comunidad bilingüe de El Paso, Texas. In: Anuario de Lingüistica Hispánica. Vol. XIV.

Medina López, Javier (1997), Lenguas en contacto. Colecção Cuadernos de Lengua Española. Dirección: L. Gómez Torrego. Madrid (Arco/Libros, S. L.).

Meeus, Baudewijn (1987), Ethnic/Language Minorities: Theoretical Issues and European Examples. In: Sociolinguistics. An International Handbook of the Science of Language and Society. Foreword by Hugo Steger and Herbert Ernst Wiegand. Preface by Ulrich Ammon, Norbert Dittmar, Klaus J. Mattheier. Edited by Ulrich Ammon, Norbert Dittmar, Klaus J. Mattheier. Vol. I. Berlin. New York (Walter de Gruyter). 
Mela Alfredo (1999), A sociologia das cidades. 1. ${ }^{a}$ edição. Tradução de Eduardo SALÓ. Colecção Temas de Sociologia. Vol. 10. Lisboa (Editorial Estampa).

Melliani, Fabienne (2000), La langue du quartier. Appropriation de l'espace et identités urbaines chez des jeunes issus de l'immigration maghrébine en banlieue rouennaise. Préface de Paul Siblot. Collection Espaces Discursifs dirigée par Thierry Bulot. Paris, Montréal, Budapest, Torino (L'Harmattan).

Mellor, Rosemary (1989), Urban Sociology: A Trend Report. In: Sociology. Vol. 23, No. 2, pp. 241-260.

Moreno Cabrera, Juan Carlos (2000), La dignidad e igualdad de las lenguas. Crítica de la discriminación lingüística. Colecção Alianza Editorial Ensayo. Vol. 154. Série El Libro Universitario. Madrid (Alianza Editorial, S. A.).

Moreno Fernández, Francisco (1990), Metodología sociolingüística. Prólogo de Manuel Alvar. Biblioteca Románica Hispánica. Série II. Estudios y Ensayos. Vol. 372. Madrid (Editorial Gredos).

Multilingual Capital. The Languages of London's Schoolchildren and their Relevance to Economic, Social and Educational Policies. Preface by The Right Honourable The Lord Mayor Alderman Clive Martin. Edited by Philip Baker and John Eversley. London (Battlebridge Publications), 2000.

Naro, Anthony J. (1992), Modelos quantitativos e tratamento estatístico. In: Introdução à sociolingüística variacionista. Colecção Cadernos Didáticos UFRJ. Vol. 4. Maria Cecilia Mollica (Org.). Apresentação de Maria Cecilia Mollica. Rio de Janeiro (Universidade Federal do Rio de Janeiro).

Navas, María Victoria (1984), Aplicación de una encuesta sociolingüística en Lisboa. Separata da Revista de Filología Románica. Vol. II.

Oksaar, Els (1996), The History of Contact Linguistics as a Discipline. In: Kontaktlinguistik. Ein internationales Handbuch zeitgenössischer Forschung. Herausgegeben von Hans Goebl, Peter H. Nelde, Źdenek Starý. 1. Halbband. Berlin, New York (Walter de Gruyter).

Pap, Leo (1949), Portuguese-American Speech. An Outline of Speech Conditions Among Portuguese Immigrants in New England and elsewhere in the United States. New York (Columbia University - King's Crown Press).

(s/d), The Portuguese-Americans. The Immigrant Heritage of America Series. Cecyle S. Neidle (ed.). Boston (Twayne Publishers. A Division of S. K. Hall and Co.).

Paquot Thierry (1994), Vive la ville! Collection Panoramiques dirigée par Guy Hennebelle et Monique Martineau. Paris (Diffusion Le Seuil).

Pederson, Lee / Billiard, Charles E. (1979), The Urban Work Sheets for the LAGS Project. In: Orbis. Tome XXVIII, $\mathrm{n}^{\circ} 1$.

Rémy Jean / Voyé Liliane (1997), A cidade: rumo a uma nova definição? 2. ${ }^{a}$ edição. Tradução de José Domingues de Almeida. Prefácio de António Custódio Gonçalves. Colecção Cidade em Questão. Vol. 9. Porto (Edições Afrontamento).

Ribeiro, Orlando (1994), A cidade e o homem. In: Opúsculos geográficos. 5. ${ }^{\circ}$ Vol. Temas urbanos. Nota introdutória de Suzanne Daveau. Lisboa (Fundação Calouste Gulbenkian). 
Sala, Marius (1985), Langues en contact: évaluation des résultats. Offprint from Studia Linguistica Diachronica et Synchronica.

(1987), Disparition des langues et contact des langues. In: Proceedings of the Fourteenth International Congress of Linguists. Edited by Werner Bahner, Joachim Schildt and Dieter Viehweger. Vol. II. Berlin/GDR, August 10-August 15. Berlin (Akademie-Verlag Berlin).

(1998), Lenguas en contacto. Segunda edición actualizada. Colecção Biblioteca Románica Hispánica. Série II. Estudios y Ensaios. Vol. 409. Madrid (Gredos).

Salvatore, Filippo (2001), Vive le Québec trilingue! In: Le Courrier de l'UNESCO. juillet/août.

Samper Padilla, José Antonio (1990), Variación lingüística y condicionantes sociales en el español de Las Palmas de Gran Canaria. Separata de Actas del Congreso de la Sociedad Española de Lingüística. XX Aniversario. Tenerife, 2-6 de abril.

(1995), Macrocorpus de la norma lingüística culta de las principales ciudades de España y América. Separata de Lingüística.

Sánchez Fernández, Manuel Jesús (2000), Apontamento para descrever o espanhol que se fala em Olivença. In: Agália. No 61.

Santarita, Paula, / Martin-Jones, Marilyn (1991), The Portuguese Speech Community. In: Multilingualism in the British Isles. Vol. 1: The Older Mother Tongues and Europe. Safder Alladina and Viv Edwards (eds.). Colecção Longman Linguistics Library. London and New York (Longman).

Sherzer, Joel / Darnell, Regna (1986), Outline Guide for the Ethnographic Study of Speech Use. In: Directions in Sociolinguistics. The Ethnography of Communication. Edited by John J. Gumperz and Dell Hymes. Preface by John J. Gumperz and Dell Hymes. Oxford and New York (Basil Blackwell).

Sibille, Jean (2000), Les langues régionales. Colecção Dominos. Vol. 220. Paris (Flammarion).

Siebenhaar, Beat (2000), Sprachvariation, Sprachwandel und Einstellung: der Dialekt der Stadt Aarau in der Labilitätszone zwischen Zürcher und Berner Mundart. Zeitschrift für Dialektologie und Linguistik, Beihefte 108. Stuttgart (Steiner).

Signorelli Amalia (1999), Antropología urbana. Traducción del italiano: Angela Giglia y Cristina Albarrán F. Prólogo de Néstor García Canclini. Epílogo de Raúl Nieto Calleja. Primera edición. Colección Autores, Textos y Temas - Antropología. Colección dirigida por $\mathrm{M}^{\mathrm{a}} \mathrm{J}$ Jesús Buxó. Barcelona, Iztapalapa (Anthropos Editorial. Rubí - División de Ciencias Sociales y Humanidades. Universidad Autónoma Metropolitana).

Silva-Corvalán, Carmen (1995), The Study of Language Contact: An Overview of the Issues. In: Spanish in Four Continents. Studies in Language Contact and Bilingualism. Edited by Carmen Silva-Corvalán. Colecção Georgetown Studies in Romance Linguistics. Héctor Campos (Series Editor). Washington (Georgetown University Press). 
Simpson, J. M. Y. (1990), The Challenge of Minority Languages. In: Minority Languages Today. A Selection from the Papers read at the First International Conference on Minority Languages held at Glasgow University from 8 to 13 September 1980. Edited by Einar Haugen, J. Derrick McClure, Derick Thomson. Edinburgh (Edinburgh University Press).

Smith, Greg (1982), Locating Populations of Minority Language Speakers: An Example of Practice from the Coventry Languages Project. L.M.P. Working Paper $\mathrm{n}^{\circ} 1$. January.

Sociolingüística andaluza 4. Encuestas del habla urbana de Sevilla. Nivel popular (1987). Vidal Lamiquiz (dir.). Miguel Ropero (ed.). Introducción de Vidal Lamiquiz. Presentación de Miguel Ropero. Serie Filosofía y Letras. Vol. 98. Sevilla (Servicio de Publicaciones de la Universidad de Sevilla).

Sociolinguistic Studies in Language Contact. Methods and Cases (1979). Edited by William Francis Mackey, Jacob Ornstein. Preface by William Francis Mackey and Jacob Ornstein. Colecção Trends in Linguistics. Série Studies and Monographs. Vol. 6. Editor: Werner Winter. The Hague, Paris, New York (Mouton Publishers).

Sornicola, Rosanna (2002), Sulla dialettologia sociologica. In: Revue de Linguistique Romane. Tome 66, $\mathrm{n}^{\circ}$ 261-262.

Stephens, Meic (1978), Linguistic Minorities in Western Europe. Llandysul, Dyfed (Gomer Press).

The ADULT LANGUAGE USE SURVEY of the Linguistic Minorities Project: The Data in Context. L.M.P./CLE Working Paper $\mathrm{n}^{\circ}$ 9. Compiled by Anna Morawska and Greg Smith on the basis of the Report by the Linguistic Minorities Project for the Department of Education and Science (July 1983) with the collaboration of Xavier Couillaud. June 1984.

The Balancing Act. Combining Symbolic and Statistical Approaches to Language (1996). Edited by Judith Klavans and Philip Resnik. Language, Speech and Communication Series. Cambridge (The MIT Press).

The MOTHER TONGUE TEACHING DIRECTORY SURVEY of the Linguistic Minorities Project. L.M.P./CLE Working Paper $n^{\circ}$ 6. Compiled by Verity Saifullah Khan, Euan Reid and Xavier Couillaud on the basis of the Report by the Linguistic Minorities Project for the Department of Education and Science (July 1983). January 1984.

The Portuguese in Canada. From the Sea to the City (2000). Foreword by John Warkentin. Edited by Carlos Teixeira and Victor M. P. da Rosa. Toronto, Buffalo, London (University of Toronto Press).

Thomason, Sarah Grey / Kaufman, Terrence (1991) Language Contact, Creolization, and Genetic Linguistics. Berkeley, Los Angeles, Oxford (University of California Press).

Truchot, Claude (1994), Les conditions du plurilinguisme en Europe. In Les langues dans l'Europe de demain. Avant-propos de Huguette Fugier, J.-M. Odéric Delefosse, André Rousseau. Sous la direction de Fernand Carton et J.-M. Odéric Delefosse. Toulouse (Association des Linguistes de l'Enseignement Supérieur - Presses de la Sorbonne Nouvelle).

Van de Craen, Piet / Beardsmore, Hugo Baetens (1987), Research on City Language. In: Sociolinguistics. An International Handbook of the Science of Language and Society. Foreword by Hugo Steger and Herbert Ernst Wiegand. Preface by Ulrich 
Ammon, Norbert Dittmar, Klaus J. Mattheier. Edited by Ulrich Ammon, Norbert Dittmar, Klaus J. Mattheier. Vol. I. Berlin. New York (Walter de Gruyter).

Varro, Gabrielle (1992), Les 'langues immigrées' face à l'école française. In: Language Problems and Language Planning. Vol. 16, $\mathrm{n}^{\circ} 2$.

Verbunt, Gilles (2001), La société interculturelle. Vivre la diversité humaine. Paris (Éditions du Seuil).

Ville, exclusion et citoyenneté. Entretiens de la ville. II (1993). Sous la direction de Joël Roman. Préface de Jean-Marie Delarue. Série Société. Paris (Éditions Esprit).

Villena Ponsoda, Juan Andrés (1994), La ciudad lingüística. Fundamentos críticos de la sociolingüística urbana. Colecção Series Linguística. Vol. II. Granada (Publicaciones de la Cátedra de História de la Lengua Española - Universidad de Granada).

Weinreich, Uriel (1979), Languages in contact. Findings and Problems. 9 $^{\text {th }}$ Printing. With a Preface by André Martinet. The Hague, Paris, New York (Mouton Publishers).

Whyte, William Foote (1996), Street Corner Society. La structure sociale d'un quartier italo-américain. Traduction de l'américain de S. Guth, J. Sevry, M. et J. Destrade, D. Vazeilles. Préface de Henri Peretz. Textes à l'appui. Série Sociologie dirigée par Michel Wieviorka. Paris (Éditions La Découverte).

Williams, Lynn (1987), Aspectos sociolingüísticos del habla de la ciudad de Valladolid. Colecção Lingüística y Filología. No 4 . Valladolid (Secretariado de Publicaciones Universidad de Valladolid).

Wilson, John (1987), The Sociolinguistic Paradox: Data as a Methodological Product. In: Language \& Communication. Vol. $7, \mathrm{n}^{\circ} 2$.

Wirth, L. (1938), Urbanism as a Way of Life. In; American Journal of Sociology. Vol. 44.

Wölck, Wolfgang (1976), Community Profiles: An Alternative Approach to Linguistic Informant Selection. In: International Journal of the Sociology of Language, $\mathrm{n}^{\circ} 9$.

Wolfram, Walt (1974), Sociolinguistic Aspects of Assimilation. Puerto Rican English in New York City. Urban Language Series. Roger W. Shuy (General Editor). Introduction to the Series by Roger W. Shuy. Vol. 9. New York (Center for Applied Linguistics).

Wolfram, Walter A. (1969), A Sociolinguistic Description of Detroit Negro Speech. Urban Language Series. Roger W. Shuy (General Editor). Introduction to the Series by Roger W. Shuy. Vol. 5. New York (Center for Applied Linguistics).

Ziegler, Arne (1996), Deutsche Sprache in Brasilien: Untersuchungen zum Sprachwandel und zum Sprachgebrauch der deutschstämmigen Brasilianer in Rio Grande do Sul. Colecção Kultur der Deutschen im Ausland. Vol. 2. Essen (Verlag Die Blaue Eule). 